\title{
A Noise Adaptive Approach to Impulse Noise Detection and Reduction
}

\author{
Isma Irum, Muhammad Sharif, Mussarat Yasmin, Mudassar Raza, and Faisal Azam \\ COMSATS Institute of Information Technology, Wah Pakistan \\ e-mail:mussaratyasmin@comsats.edu.pk
}

\begin{abstract}
A noise adaptive filter has been proposed in this study aiming to estimate the original image pixel values in the presence of impulse noise in monochromatic images. The proposed filter approach is noise adaptive that as the percentage of noise density increases in the image, the size of neighborhood in filtering window is also increased. Proposed approach comprises of two stages, one is impulse noise detection and the other is impulse noise reduction or cancellation. First stage is based on median and mean distance and thresholding whereas the second stage is based on reconstruction of the image using the values of neighboring pixels of the pixel under consideration detected as contaminated pixel by first stage. Reconstruction is done by estimating reference values using uncorrupted pixels in the neighborhood of pixel under consideration. The proposed method has been compared to various existing methods by using peak signal to noise ratio (PSNR) for measuring the objective quality strength. To measure the impulse noise detection the method has also been compared with other existing methods using the ratio of mis detection (MD) and false detection (FD).
\end{abstract}

Key words: image enhancement, image restoration, random impulse noise, Salt \& Pepper noise, switching trimmed median filter

\section{Introduction}

The process of image acquisition and transmission often corrupts the digital images by impulse noise. Impulse noise contaminates the pixels of original image with fixed values of allowable minimum and maximum intensities (salt \& pepper noise) or with a range of minimum and maximum intensities (random impulse noise). Any impulse noise seriously affects the quality of image while distorting the image details, features, edges, spatial resolutions etc. by adding false details to the image. Therefore it is very important and essential to remove noise from the image for the implementation of more image processing operations like edge detection, image segmentation, object detection, image features extraction, etc.

Image denoising has been a hot research area since the last decades and a huge number of techniques have been proposed for noise removal. These techniques differ from each other due to the difference of nature of noise. For impulse noise standard median filter is the most popular and common technique having the drawback of treating contaminated and uncorrupted pixels at the same time and propagating this error pixel by pixel throughout the image. To overcome this drawback switching mechanism has been introduced that selects only the contaminated pixel for filtration process and gives better performance (Tripathi et al. 2011).

Weighted median filters (WMF) is another approach that assigns weights to the pixels existing in filtering window where the central pixel constitutes the higher weight(Chan et al. 2004, Chen \& Wu 2001).

Switching filter consists of impulse noise detection as a main process, many approaches have been proposed for impulse noise detection. Some of these approaches are based on local image statistics that considers the measurement in change of intensity in a pixel from its neighborhood; these image statistics may include ranked-ordered differences (Garnett et al. 2005). Some other techniques including boundary discriminative noise detection (BDND) ( $\mathrm{Ng} \& \mathrm{Ma} 2006)$, one 
dimensional Laplacian operator (Zhang \& Karim 2002), neural network and fuzzy logic based techniques (Toprak \& Güler 2006; Zvonarev et al. 2005) are also helpful in the detection of impulse noise. Some other techniques are noise adaptive which means that the size of neighborhood changes with the change in noise density (Eng \& Ma 2001, Lee et al. 2007, Vijaykumar et al. 2008).

A noise adaptive approach to impulse noise detection and reduction has been proposed in this study. The proposed method detects noise on the basis of thresholding based distance from median and mean of pixel under consideration. The image is reconstructed by computing the median of reference values constructed from the uncorrupted pixels existing in the neighborhood of contaminated pixel. The structure of upcoming sections in this paper is organized as: Section 2 contains detailed study of proposed work including noise models, impulse noise detection and filtration process. Section 3 gives a discussion on results in detail and Section 4 concludes the whole paper. In the end section 5 lists the references used in this paper.

\section{Methodology}

Consider a contaminated input image i.e., $C=\{c(m, n) \mid 1 \leq m \leq H, 1 \leq n \leq W\}$ of size $H \times W$ pixels. $c(m, n) \in\left[0, Z^{+}\right]$and $C$ has a dynamic range $\left[c_{M N}, c_{L_{x}}\right]$. The objective of proposed approach is to generate a noiseless image $A=\{a(m, n) \mid 1 \leq m \leq H, 1 \leq n \leq W\}$ of $\quad$ size pixels $a(m, n) \in\left[0, Z^{+}\right]$and A having a dynamic range and better visual quality than $C$, where $a_{F 1}>c_{\text {min }}$ and $a_{F 2}<c_{\text {max }}$.

\section{Noise models}

Four impulse noise models (Ng \& Ma 2006) have been implemented in this study for the purpose of examining the performance of proposed impulse noise detection and cancellation method. The contaminated pixels do have the intensity values equal to or near to the minimum and maximum values of allowed dynamic range. For a noiseless original image a pixel at location is denoted by and the corresponding pixel in contaminated image is denoted by.

\section{Noise model 1}

Probability distribution function for noise model 1 is given by

$$
f(c)= \begin{cases}\frac{d}{2} & \text { for } c=\text { Min } \\ 1-d & \text { for } c=a_{m, n} \\ \frac{d}{2} & \text { for } c=\text { Max }\end{cases}
$$

Here contaminated image contains fixed values of Max and Min i.e., 255 and 0 for salt and pepper noise respectively. is noise density in the image having equal probabilities for salt and pepper noise.

\section{Noise model 2}

Probability distribution function for noise model 2 is given by

$f(c)= \begin{cases}d_{1} & \text { for } c=\text { Min } \\ 1-d & \text { for } c=a_{m, n} \\ d_{2} & \text { for } c=\text { Max }\end{cases}$

Here contaminated image contains fixed values of and i.e., 255 and 0 for salt and pepper noise respectively but with unequal probabilities. is noise density in the image and

\section{Noise model 3}

Probability distribution function for noise model 3 is given by

$$
f(c)= \begin{cases}\frac{d}{2 P_{1}} & \text { for } M n \leq c \leq M n+P_{1} \\ 1-d & \text { for } c=a_{m, n} \\ \frac{d}{2 P_{2}} & \text { for } M a x-P_{2} \leq c \leq M a x\end{cases}
$$

Here contaminated image contains dynamic values for salt i.e., $M a x-P_{2}$ to $M a x$ and for pepper i.e., Min to $M i n+P_{1}$ with equal probability. $d$ is noise density in the image. 
Isma Irum et al./A Noise Adaptive Approach to Impulse.......

\section{Noise model 4}

Probability distribution function for noise model 4 is given by

$$
f(c)= \begin{cases}\frac{d_{1}}{P_{1}} & \text { for } M i n \leq c \leq M i n+P_{1} \\ 1-d & \text { for } c=a_{m, n} \\ \frac{d_{2}}{P_{2}} & \text { for } M a x-P_{2} \leq c \leq M a x\end{cases}
$$

Here contaminated image contains dynamic values for salt i.e., $M a x-P_{2}$ to $M a x$ and for pepper i.e., $M i n$ to $M n+P_{1}$ but with unequal probabilities $d=d_{1}+d_{2}$. is noise density in the image and $d_{1} \neq d_{2}$.

\section{Impulse noise detection}

To detect whether a pixel under consideration is a contaminated one or not, two distance formulae have been used; one is the distance of contaminated pixel from median of its uncorrupted neighboring pixels and the other is the distance of contaminated pixel from mean of its uncorrupted neighboring pixels. As proposed approach is noise adaptive, one means size of neighborhood varies with noise densities in the image. Following sizes have been adopted by the proposed approach according to change in noise densities.

$$
f((2 K+1) \times(2 K+1))=\left\{\begin{array}{lll}
K=1 & (3 \times 3) & \text { for } 10 \leq d \leq 0 \\
K=2 & (5 \times 0) & \text { for } 30<d \leq 60 \\
K=3 & (7 \times 7) & \text { for } 00<d \leq 90
\end{array}\right.
$$

Distances are defined by the following equations

$$
\begin{aligned}
& T_{1}=\left\{\begin{array}{c}
\left|c_{m, n}-\operatorname{median}\left(a_{n-K+, n-K+j}\right)\right| \\
\text { for } j=0 \ldots 2 K \\
\text { for } j=0 \ldots 2 K
\end{array}\right. \\
& T_{2}=\left\{\begin{array}{c}
\left|c_{m, n}-\operatorname{mean}\left(a_{m-K+i, n+K}\right)\right| \\
\text { for } j=0 \ldots 2 K \\
\text { for } j=0 \ldots 2 K
\end{array}\right.
\end{aligned}
$$

By using the expression 6 and expression 7 a noise map is generated for marking the contaminated pixels.

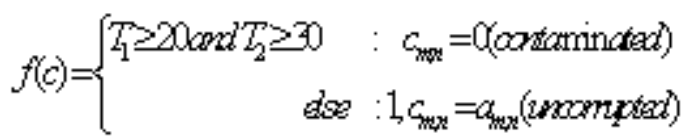

Examples of noise map and filtering windows have been shown in Figure1, ' 0 ' shows the contaminated pixel and ' 1 ' shows the uncorrupted pixel in the noise map.

\begin{tabular}{|l|l|l|}
\hline 1 & 1 & 1 \\
\hline 1 & 0 & 1 \\
\hline 1 & 1 & 0 \\
\hline
\end{tabular}

$$
\begin{array}{|l|l|l|}
\hline a_{11} & a_{12} & a_{13} \\
\hline a_{21} & C_{22} & a_{23} \\
\hline a_{31} & a_{32} & C_{33} \\
\hline
\end{array}
$$

Figl(a) Noise Map Figl(b) $K=1(3 \times 3)$ Filtering window

\begin{tabular}{|l|l|l|l|l|}
\hline 1 & 1 & 1 & 1 & 1 \\
\hline 1 & 1 & 1 & 1 & 1 \\
\hline 1 & 1 & 0 & 1 & 1 \\
\hline 0 & 1 & 1 & 1 & 0 \\
\hline 1 & 1 & 1 & 0 & 1 \\
\hline
\end{tabular}

Figl(C) Noise Map

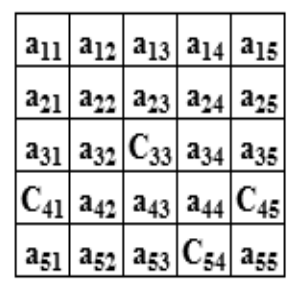

Figl(d) $K=2$ (5X5) Filtering window

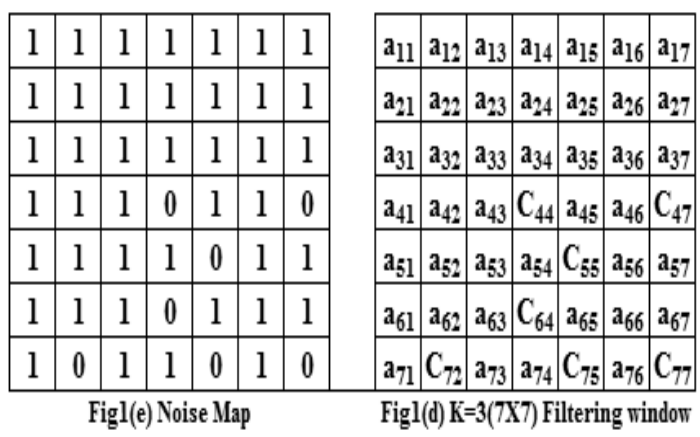

Fig.1. Examples of noise map and filtering window for $\mathrm{K}=1,2,3$

\section{Filtration process}

The contaminated image is scanned pixel by pixel from left to right and top to bottom fashion. Once a pixel is detected as a contaminated one, the size of neighborhood is selected according to expression 5 and all other pixels in the neighborhood are also passed from noise detection process. Filtration process is based on the concept of reference values obtained from uncorrupted pixels existing in selected neighborhood. The steps of the whole process are given in the following. 
1. The pixels before the under consideration central pixel are designated as Pre-neighborhood and the pixels after the central pixel are designated as Postneighborhood. Following expressions define the Pre and Post neighborhood.

$$
\begin{aligned}
& \operatorname{Pr} n=\left\{\begin{array}{l}
a_{m-K+i, n-K+j} ; \\
m-K, n-K \leq m-K+i, n-K+j \leq m, n-1
\end{array}\right. \\
& P=\left\{\begin{array}{l}
a_{m-X+i, n-X_{+j}} ; \\
m, n+1 \leq m-K+i, n-K+j \leq m+K, n+K
\end{array}\right.
\end{aligned}
$$

2. The point difference of first element of Prn from second, third, fourth and so on is calculated and similarly for Psn the point difference is calculated. The absolute sum of both differences obtained is calculated by the following expression

$$
\begin{array}{r}
S O D=\mid \operatorname{Pr} n(1)-\operatorname{Pr} n(2)-\ldots-\operatorname{Pr} n(s) \\
+ \\
P \operatorname{Sn}(1)-\operatorname{Psn}(2)-\ldots-\operatorname{Ps} n(s) \mid
\end{array}
$$

Where $s$ is the maximum number of elements in Prn and Psn

3. The reference values are obtained by dividing the SOD by resultant value (V) obtained by dividing the SOD by each uncorrupted pixel in the neighborhood. Expression 12 and expression 13 describe this step as follows

$$
\begin{aligned}
& V_{m-X+i, n-X+j}=\frac{S O D}{a_{m-k+i, n-k+j}} \\
& R_{m-X+i, n-X+j}=\frac{S O D}{V_{m-K+i, n-K+j}}
\end{aligned}
$$

where $0 \leq i, j \leq 2 K$ and $K=1,2,3$

4. Finally the central contaminated pixel is replaced by median of reference values of its neighborhood as

$c_{m, n}=\operatorname{median}\left(R_{m-X+i, n-X+j}\right)$

where $0 \leq i, j \leq 2 K$ and $K=1,2,3$

\section{Results and Discussion}

Three standard test images 'Lena', 'Baboon' and 'Peppers' have been used for verifying the performance of proposed approach as shown in Fig. 2.

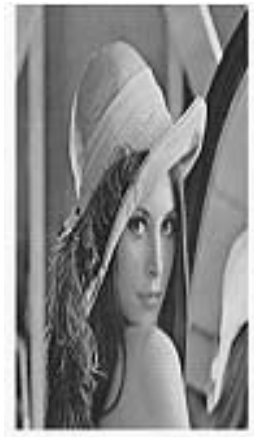

(a) Lena

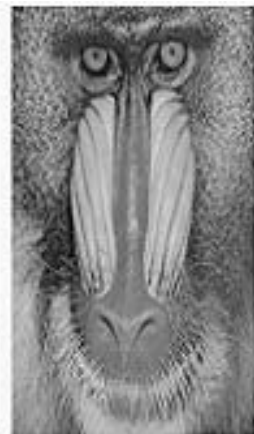

(b) Baboon

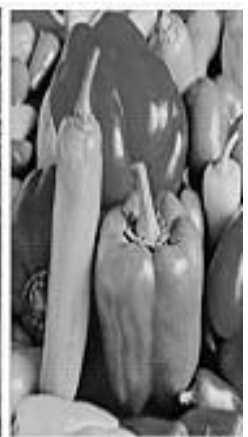

(c) Peppers
Fig.2. Standard test images

Experiments have been carried out with various noise densities varying from $10 \%$ to $90 \%$ for all the four impulse noise models. The selected techniques used for comparison are advanced boundary discriminative noise detection algorithm (ABDND) (Tripathi et al. 2011), adaptive switching median filter (ASMF) (Nallaperumal et al. 2007), boundary discriminative noise detection (BDND) ( $\mathrm{Ng} \& \mathrm{Ma} 2006$ ), Fuzzy detector (Garnett et al. 2005), iterative adaptive switching median filter (IASMF) (Luo 2007), Laplacian detector (Nallaperumal et al. 2006), morphological adaptive switching median filter (MASMF) (Zhang et al. 2008), morphological residue detector (MRD) (ZeFeng et al. 2007) and progressive switching median filter (PSMF) (Wang \& Zhang 1999). Table1 gives the best possible values of selected techniques dependent parameters (Tripathi \& Ghanekar et al. 2011).

To evaluate the performance of proposed impulse noise detector two measures miss detection (MD) and false detection (FD) have been used. Miss detection refers to a situation when a contaminated pixel is detected as uncorrupted whereas false detection refers to a situation when an uncorrupted pixel is detected as a contaminated one. This means an increased value for $\mathrm{MD}$ and FD shows the decreased performance of the detector. 
Table 1. Best possible parameter values

\begin{tabular}{l|c|c|c|c|c}
\hline Method & Noise Model & wl & w2 & u & T \\
\hline ABDND & $1,2,3,4$ & 11 & 15 & - & - \\
ASMF & $1,2,3,4$ & 3 & - & - & - \\
BDND & $1,2,3,4$ & 3 & - & - & - \\
Fuzzy & $1 \& 2$ & 30 & 25 & 2 & - \\
& $3 \& 4$ & 20 & - & - & - \\
IASMF & $1,2,3,4$ & 3 & - & - & 140 \\
Laplacian & $1 \& 2$ & - & - & - & 130 \\
MASMF & $3 \& 4$ & - & - & 25 \\
Min-Max & $1,2,3,4$ & 7 & - & - & 30 \\
MRD & $1 \& 2$ & - & - & - & - \\
PSMF & $3 \& 4$ & - & - & - \\
\hline
\end{tabular}

To evaluate the performance of proposed filtration process, image objective quality measure Peak-signalto-noise ratio has been used. PSNR is given by the following expression.

$$
P S N R=\operatorname{10log}_{10} \frac{(255)^{2}}{M S E} d B \quad \text { (15) }
$$

where

$M S E=\frac{\sum_{m=1}^{H} \sum_{n=1}^{W}(c(m, n)-A(m, n))^{2}}{H \times W}$
For the 'Lena' image comparative results for MD and FD have been given in Table 2, 4, 6 and 8 respectively for four noise models discussed in section 2 whereas the comparative results for PSNR have been given in Table 3, 5, 7 and Table 9 respectively. The results have also been obtained for 'Baboon' and 'Peppers' images which are very similar to the results of 'Lena' image but due to the space limitations those have not been included here.

Table 2. Comparitive results for number of MD \& FD on Lena image according to noise model 1

\begin{tabular}{|c|c|c|c|c|c|c|c|c|c|c|c|c|}
\hline \multicolumn{2}{|c|}{$\begin{array}{l}\text { Noise } \\
\text { Density } \%\end{array}$} & \multirow{2}{*}{\begin{tabular}{|c} 
ABDND \\
0
\end{tabular}} & \multirow{2}{*}{$\frac{\text { ASTIF }}{0}$} & \multirow{2}{*}{$\begin{array}{c}\text { BDND } \\
0\end{array}$} & \multirow{3}{*}{$\begin{array}{c}\text { Fursy } \\
0 \\
2854\end{array}$} & \multirow{3}{*}{$\begin{array}{c}\text { LASHF } \\
0 \\
10\end{array}$} & \multirow{3}{*}{$\begin{array}{c}\begin{array}{r}\text { Method } \\
\text { Laplacian }\end{array} \\
126 \\
431\end{array}$} & \multirow{3}{*}{$\begin{array}{c}\text { MASMF } \\
0 \\
231\end{array}$} & \multirow{3}{*}{$\begin{array}{c}\text { Min: } \\
\text { Max } \\
0 \\
10\end{array}$} & \multirow{3}{*}{$\begin{array}{c}\text { MRD } \\
0 \\
13\end{array}$} & \multirow{3}{*}{$\begin{array}{c}\text { PSHF } \\
487 \\
1873\end{array}$} & \multirow{3}{*}{\begin{tabular}{|c} 
Proposed \\
0 \\
8
\end{tabular}} \\
\hline 10 & $\mathrm{MD}$ & & & & & & & & & & & \\
\hline & $\mathrm{FD}$ & 10 & 14 & 7 & & & & & & & & \\
\hline \multirow[t]{2}{*}{20} & $\mathrm{MD}$ & 0 & 0 & 0 & 0 & 0 & 557 & 0 & 0 & 0 & 882 & 0 \\
\hline & $\mathrm{FD}$ & 1 & 0 & 4 & 2485 & 14 & 741 & 21 & 1 & 1 & 1814 & 0 \\
\hline \multirow[t]{2}{*}{30} & $\mathrm{MD}$ & 0 & 0 & 0 & 0 & 0 & 1413 & 0 & 0 & 0 & 1394 & 0 \\
\hline & $\mathrm{FD}$ & 0 & 0 & 4 & 1912 & 31 & 1279 & 0 & 0 & 0 & 1774 & 0 \\
\hline \multirow[t]{2}{*}{40} & $\mathrm{MD}$ & 0 & 0 & 0 & 4 & 0 & 2697 & 0 & 0 & 0 & 1862 & 0 \\
\hline & $\mathrm{FD}$ & 0 & 0 & 5 & 1317 & 41 & 1940 & 0 & 0 & 0 & 1841 & 0 \\
\hline \multirow[t]{2}{*}{50} & $\mathrm{MD}$ & 0 & 0 & 0 & 11 & 0 & 4107 & 0 & 0 & 0 & 2323 & 0 \\
\hline & $\mathrm{FD}$ & 0 & 0 & 8 & 765 & 35 & 2391 & 0 & 0 & 0 & 1876 & 0 \\
\hline \multirow[t]{2}{*}{60} & $\mathrm{MD}$ & 0 & 0 & 0 & 34 & 0 & 6311 & 0 & 0 & 0 & 2921 & 0 \\
\hline & $\mathrm{FD}$ & 0 & 0 & 6 & 518 & 66 & 2595 & 0 & 0 & 0 & 2239 & 0 \\
\hline \multirow[t]{2}{*}{70} & $\mathrm{MD}$ & 0 & 0 & 0 & 69 & 0 & 8860 & 0 & 0 & 0 & 3756 & 0 \\
\hline & $\mathrm{FD}$ & 0 & 0 & 7 & 272 & 65 & 2348 & 0 & 0 & 0 & 2907 & 0 \\
\hline \multirow[t]{2}{*}{80} & $\mathrm{MD}$ & 0 & 0 & 0 & 110 & 0 & 11192 & 0 & 0 & 0 & 6754 & 0 \\
\hline & $\mathrm{FD}$ & 0 & 0 & 9 & 133 & 55 & 1923 & 0 & 0 & 0 & 3530 & 0 \\
\hline \multirow[t]{2}{*}{90} & $\mathrm{MD}$ & 0 & 0 & 0 & 197 & 0 & 13394 & 0 & 0 & 0 & 15851 & 0 \\
\hline & $\mathrm{FD}$ & 0 & 0 & 65 & 72 & 48 & 1089 & 0 & 0 & 0 & 3442 & 0 \\
\hline
\end{tabular}


Nepal Journal of Science and Technology Vol. 15, No.1 (2014) 67-76

Table 3. Comparitive results for PSNR of Lena image according to noise model 1

\begin{tabular}{l|l|l|l|l|l|l|l|l|l|l|l}
\hline $\begin{array}{l}\text { Noise } \\
\begin{array}{l}\text { Density, } \\
\%\end{array}\end{array}$ & ABDND & ASMF & BDND & Fuczy & IASMF & Laplacian & MASMF & $\begin{array}{l}\text { Min- } \\
\text { Max }\end{array}$ & MRD & PSMF & Proposed \\
\hline 10 & 38.92 & 3892 & 38.92 & 33.98 & 38.92 & 32.95 & 3835 & 3892 & 38.92 & 27.89 & 41.12 \\
20 & 34.69 & 3469 & 34.69 & 32.96 & 34.69 & 31.50 & 3469 & 3409 & 34.69 & 27.37 & 36.89 \\
30 & 32.60 & 3260 & 32.60 & 31.21 & 32.99 & 26.20 & 3260 & 3260 & 32.60 & 26.34 & 34.80 \\
40 & 30.63 & 30.63 & 30.63 & 29.84 & 30.13 & 21.39 & 30.63 & 3063 & 30.63 & 25.66 & 32.83 \\
50 & 28.98 & 2898 & 28.98 & 28.12 & 28.97 & 17.64 & 2898 & 2898 & 28.98 & 23.95 & 31.18 \\
60 & 27.50 & 2750 & 27.50 & 26.65 & 27.48 & 15.44 & 2750 & 2750 & 27.50 & 20.89 & 29.70 \\
70 & 26.18 & 26.18 & 26.18 & 24.58 & 26.17 & 11.47 & 26.18 & 26.18 & 26.18 & 15.83 & 28.38 \\
80 & 24.66 & 2466 & 24.66 & 21.50 & 24.63 & 8.01 & 2466 & 2466 & 24.66 & 10.62 & 26.86 \\
90 & 22.47 & 22.47 & 22.28 & 18.18 & 22.41 & 6.60 & 22.47 & 22.47 & 22.47 & 692 & 24.67 \\
\hline
\end{tabular}

Table 4. Comparitive results for number of MD \& FD on Lena image according to noise model 2

\begin{tabular}{c|c|c|c|c|c|c|c|c|c|c|c|c}
\hline \multicolumn{1}{l}{$\begin{array}{l}\text { Noise } \\
\text { Density \% }\end{array}$} & ABDND & ASMF & BDND & Fur M & LASMF & Laplacian & MASMF & Min & MRD & PSMF & Proposed \\
\hline 10 & MD & 0 & 0 & 0 & 0 & 0 & 141 & 0 & 0 & 0 & 589 & 0 \\
$6+4$ & FD & 18 & 18 & 3 & 2971 & 13 & 501 & 220 & 19 & 84 & 1859 & 4 \\
20 & MD & 0 & 0 & 0 & 0 & 0 & 598 & 0 & 0 & 0 & 712 & 0 \\
$8+12$ & FD & 1 & 0 & 3 & 2590 & 16 & 817 & 38 & 0 & 0 & 1834 & 0 \\
30 & MD & 0 & 0 & 0 & 6 & 0 & 1982 & 0 & 0 & 0 & 1999 & 0 \\
$10+20$ & FD & 0 & 0 & 6 & 2235 & 45 & 1715 & 11 & 0 & 0 & 2001 & 0 \\
40 & MD & 0 & 0 & 0 & 10 & 0 & 3411 & 0 & 0 & 0 & 2744 & \\
$25+15$ & FD & 0 & 0 & 15 & 1485 & 67 & 2195 & 1 & 0 & 0 & 2016 & 0 \\
50 & MD & 0 & 0 & 0 & 23 & 0 & 4873 & 0 & 0 & 0 & 1601 & 0 \\
$20+30$ & FD & 0 & 0 & 7 & 945 & 85 & 2747 & 0 & 0 & 0 & 2314 & 0 \\
60 & MD & 0 & 0 & 0 & 57 & 0 & 7168 & 0 & 0 & 0 & 2243 & 0 \\
$25+35$ & FD & 0 & 0 & 10 & 558 & 91 & 2956 & 0 & 0 & 0 & 2887 & 0 \\
70 & MD & 0 & 0 & 0 & 114 & 0 & 9550 & 0 & 0 & 0 & 6415 & 0 \\
$40+30$ & FD & 0 & 0 & 16 & 305 & 82 & 2464 & 0 & 0 & 0 & 3526 & 0 \\
80 & MD & 0 & 0 & 0 & 171 & 0 & 12293 & 0 & 0 & 0 & 9747 & 0 \\
$35+45$ & FD & 0 & 0 & 66 & 170 & 84 & 1967 & 0 & 0 & 0 & 4749 & 0 \\
90 & MD & 0 & 0 & 0 & 294 & 0 & 14210 & 0 & 0 & 0 & 19329 & 0 \\
$40+50$ & FD & 0 & 0 & 618 & 64 & 56 & 1234 & 0 & 0 & 0 & 4003 & 0 \\
\hline
\end{tabular}

Table 5. Comparative results for PSNR of Lena image according to noise model 2

\begin{tabular}{|c|c|c|c|c|c|c|c|c|c|c|c|}
\hline \multirow{2}{*}{$\begin{array}{c}\text { Noise } \\
\text { Density, } \\
\%\end{array}$} & \multicolumn{11}{|c|}{ Method } \\
\hline & ABDND & ASHF & BDND & Fursy & LASHF & Lap lacian & MASMF & $\begin{array}{l}\operatorname{Min}_{\mathrm{Max}} \\
\text { Man }\end{array}$ & MRD & PSMF & Prosposed \\
\hline $\begin{array}{c}10 \\
6+4\end{array}$ & 38.92 & 38.92 & 38.92 & 33.70 & 38.92 & 3091 & 37.47 & 38.92 & 38.91 & 28.01 & 40.82 \\
\hline $\begin{array}{c}20 \\
8+12\end{array}$ & 34.84 & 34.84 & 34.84 & 32.67 & 34.84 & 2481 & 34.12 & 34.84 & 34.84 & 27.33 & 36.74 \\
\hline $\begin{array}{c}30 \\
10+20\end{array}$ & 32.78 & 32.78 & 32.78 & 30.72 & 32.72 & 1851 & 31.61 & 32.78 & 32.78 & 25.83 & 34.68 \\
\hline $\begin{array}{c}40 \\
25+15\end{array}$ & 30.19 & 30.19 & 30.19 & 29.19 & 30.18 & 15.41 & 30.19 & 30.19 & 30.19 & 24.58 & 32.09 \\
\hline $\begin{array}{c}50 \\
20+30\end{array}$ & 28.80 & 28.80 & 28.80 & 27.99 & 28.79 & 13.48 & 28.80 & 28.80 & 28.80 & 23.60 & 30.70 \\
\hline $\begin{array}{c}60 \\
25+35\end{array}$ & 27.81 & 27.81 & 27.81 & 25.55 & 27.76 & 1135 & 27.81 & 27.81 & 27.81 & 19.41 & 29.71 \\
\hline $\begin{array}{c}70 \\
40+30\end{array}$ & 25.99 & 25.99 & 25.99 & 23.17 & 25.97 & 9.25 & 25.98 & 25.99 & 25.99 & 13.63 & 27.89 \\
\hline $\begin{array}{c}\infty \\
35+45\end{array}$ & 24.48 & 24.48 & 24.48 & 21.60 & 24.46 & 7.89 & 24.48 & 24.48 & 24.48 & 9.53 & 26.38 \\
\hline $\begin{array}{c}90 \\
40+50\end{array}$ & 22.49 & 22.49 & 22.48 & 17.52 & 22.17 & 6.61 & 22.49 & 22.49 & 22.49 & 6.40 & 24.39 \\
\hline
\end{tabular}


Isma Irum et al./A Noise Adaptive Approach to Impulse........

Table 6. Comparative results for number of MD and FD on Lena image according to noise model 3

\begin{tabular}{|c|c|c|c|c|c|c|c|c|c|c|c|c|}
\hline \multirow{2}{*}{\multicolumn{2}{|c|}{$\begin{array}{c}\text { Noise } \\
\text { Density, } \\
(\%)\end{array}$}} & \multicolumn{11}{|c|}{ Method } \\
\hline & & ABDHD & $A S M F$ & BDND & Fuzzy & IASMF & Laplacian & $M A S M F$ & $\begin{array}{l}\text { Min- } \\
\text { Max }\end{array}$ & MRD & FSMF & $\begin{array}{l}\text { Prop } \\
\text { osed }\end{array}$ \\
\hline 10 & $\mathrm{MD}$ & 0 & 4255 & 0 & 120 & 5840 & 170 & 3726 & 1085 & 1785 & 530 & 0 \\
\hline & FD & 270 & 19 & 7 & 10017 & 3 & 534 & 229 & 3316 & 1102 & 1890 & \\
\hline 20 & $\mathrm{MD}$ & 0 & 10144 & 38 & 813 & 11657 & 692 & 9615 & 3885 & 7598 & 1054 & 0 \\
\hline & FD & 134 & 0 & 7 & 10171 & 0 & 873 & 27 & 2790 & 50 & 1892 & \\
\hline 30 & $\mathrm{MD}$ & 0 & 15791 & 192 & 2264 & 17300 & 1385 & 15911 & 7499 & 13800 & 1527 & 0 \\
\hline & $\mathrm{FD}$ & 103 & 0 & 7 & 8521 & 0 & 1537 & 2 & 2092 & 4 & 1787 & \\
\hline 40 & $\mathrm{MD}$ & 0 & 21325 & 712 & 4782 & 23248 & 2586 & 22083 & 12246 & 20206 & 2058 & 0 \\
\hline & $\mathrm{FD}$ & 93 & 0 & 23 & 6342 & 0 & 2170 & 0 & 1297 & 0 & 1753 & \\
\hline 50 & $\mathrm{MD}$ & 0 & 27324 & 1859 & 7931 & 29053 & 4241 & 27963 & 17368 & 25907 & 2662 & 0 \\
\hline & FD & 66 & 0 & 21 & 4552 & 0 & 2771 & 0 & 896 & 0 & 1800 & \\
\hline 60 & $\mathrm{MD}$ & 0 & 32775 & 3670 & 11848 & 34984 & 6244 & 33991 & 23058 & 32344 & 3221 & 0 \\
\hline & $\mathrm{FD}$ & 55 & 0 & 13 & 3038 & 0 & 2803 & 0 & 447 & 0 & 2134 & \\
\hline 70 & $\mathrm{MD}$ & 0 & 38710 & 6762 & 16539 & 40448 & 8574 & 40036 & 28735 & 38692 & 4360 & 0 \\
\hline & FD & 45 & 0 & 26 & 1953 & 0 & 2712 & 0 & 254 & 0 & 2897 & \\
\hline 80 & $\mathrm{MD}$ & 0 & 44010 & 10431 & 21536 & 46270 & 10719 & 46411 & 34785 & 42932 & 7410 & 0 \\
\hline & FD & 30 & 0 & 41 & 1164 & 0 & 2175 & 0 & 129 & 0 & 3604 & \\
\hline 90 & $\mathrm{MD}$ & 0 & 49573 & 15975 & 27194 & 50223 & 12828 & 52401 & 40689 & 44932 & 16779 & 0 \\
\hline & $\mathrm{FD}$ & 15 & 0 & 109 & 506 & 0 & 1266 & 0 & 51 & 0 & 3312 & \\
\hline
\end{tabular}

Table 7. Comparative results for PSNR of Lena image according to noise model 3

\begin{tabular}{|c|c|c|c|c|c|c|c|c|c|c|c|}
\hline \multirow{2}{*}{$\begin{array}{l}\text { Noise } \\
\text { Density, } \\
\text { (\%) }\end{array}$} & \multicolumn{11}{|c|}{ Method } \\
\hline & ABDND & AS MF & BDWD & Fuzzy & IAS MF & Laplacian & MAS MF & $\begin{array}{l}\text { Min- } \\
\text { M ax }\end{array}$ & MRD & PS MF & Proposed \\
\hline 10 & 37.49 & 16.78 & 3752 & 2727 & 1594 & 32.29 & 17.71 & 21.59 & 20.43 & 28.01 & 39.52 \\
\hline 20 & 34.03 & 13.01 & 3397 & 2081 & 1288 & 27.54 & 13.64 & 15.90 & 14.49 & 27.51 & 36.03 \\
\hline 30 & 31.85 & 11.10 & 2952 & 1663 & 11.15 & 22.99 & 11.47 & 13.07 & 11.91 & 26.60 & 33.85 \\
\hline 40 & 30.25 & 9.69 & 2601 & 13.78 & 9.89 & 19.51 & 10.07 & 11.06 & 10.30 & 25.53 & 32.25 \\
\hline 50 & 28.57 & 8.66 & 2566 & 1152 & 8.87 & 16.49 & 9.06 & 9.62 & 9.12 & 24.87 & 30.57 \\
\hline 60 & 27.21 & 7.73 & 2261 & 9.65 & 8.02 & 13.83 & 8.10 & 8.45 & 8.14 & 22.31 & 29.21 \\
\hline 70 & 25.99 & 7.08 & 1822 & 8.40 & 7.28 & 11.27 & 7.39 & 7.58 & 7.39 & 18.37 & 27.99 \\
\hline 80 & 24.01 & 6.42 & 1199 & 7.30 & 6.67 & 9.41 & 6.83 & 6.78 & 6.78 & 11.98 & 26.01 \\
\hline 90 & 21.79 & 5.89 & 8.63 & 6.41 & 6.02 & 7.60 & 6.21 & 6.22 & 6.20 & 7.33 & 23.79 \\
\hline
\end{tabular}

Table 8. Comparative results for number MD and FD of Lena image according to noise model 4

\begin{tabular}{|c|c|c|c|c|c|c|c|c|c|c|c|c|}
\hline \multirow{2}{*}{\multicolumn{2}{|c|}{$\begin{array}{c}\text { Hoise } \\
\text { Dersity, (\%) }\end{array}$}} & \multicolumn{11}{|c|}{ Method } \\
\hline & & \multirow{2}{*}{$\begin{array}{c}\mathrm{ABDHD} \\
\frac{0}{288}\end{array}$} & \multirow{2}{*}{$\begin{array}{c}\text { ASMF } \\
4362 \\
19\end{array}$} & \multirow{2}{*}{$\begin{array}{c}\text { BDHD } \\
0 \\
7\end{array}$} & \multirow{2}{*}{$\begin{array}{c}\text { Fuzy } \\
138 \\
10048\end{array}$} & \multirow{2}{*}{$\begin{array}{c}\text { IASMF } \\
5765 \\
0\end{array}$} & \multirow{2}{*}{$\begin{array}{c}\text { Laplaciam } \\
205 \\
554\end{array}$} & \multirow{2}{*}{$\begin{array}{c}\text { MAS MF } \\
225 \\
4079\end{array}$} & \multirow{2}{*}{$\begin{array}{c}\text { Mirt } \\
\text { Max }\end{array}$} & \multirow{2}{*}{$\begin{array}{l}\text { MRD } \\
1779 \\
1238\end{array}$} & \multirow{2}{*}{$\begin{array}{c}\text { PSMF } \\
667 \\
1855\end{array}$} & \multirow{2}{*}{$\begin{array}{c}\text { Proposed } \\
0\end{array}$} \\
\hline $\begin{array}{c}10 \\
6+4\end{array}$ & $\begin{array}{l}\mathrm{MD} \\
\mathrm{FD}\end{array}$ & & & & & & & & & & & \\
\hline $\begin{array}{c}20 \\
8+12\end{array}$ & $\begin{array}{l}\mathrm{MD} \\
\mathrm{FD}\end{array}$ & $\begin{array}{c}0 \\
149\end{array}$ & $\begin{array}{c}10134 \\
0\end{array}$ & $\begin{array}{l}35 \\
14\end{array}$ & $\begin{array}{c}904 \\
10253\end{array}$ & $\begin{array}{c}11497 \\
0\end{array}$ & $\begin{array}{l}616 \\
900\end{array}$ & $\stackrel{23}{9753}$ & $\begin{array}{l}3862 \\
2948\end{array}$ & $\begin{array}{c}7027 \\
67\end{array}$ & $\begin{array}{l}810 \\
1900\end{array}$ & 0 \\
\hline $\begin{array}{c}30 \\
10+20\end{array}$ & $\begin{array}{l}\mathrm{MD} \\
\mathrm{FD}\end{array}$ & $\begin{array}{c}0 \\
100\end{array}$ & $\begin{array}{c}15920 \\
0\end{array}$ & $\begin{array}{c}385 \\
30\end{array}$ & $\begin{array}{l}2732 \\
8 \infty 0\end{array}$ & $\begin{array}{c}17281 \\
0\end{array}$ & & $\begin{array}{c}12 \\
21965\end{array}$ & $\begin{array}{l}7764 \\
2449\end{array}$ & $\begin{array}{c}13676 \\
22\end{array}$ & $\begin{array}{l}2283 \\
1968\end{array}$ & 0 \\
\hline $\begin{array}{c}40 \\
25+15\end{array}$ & $\begin{array}{l}\mathrm{MD} \\
\mathrm{FD}\end{array}$ & $\begin{array}{c}0 \\
86\end{array}$ & $\begin{array}{c}21417 \\
0\end{array}$ & $\begin{array}{c}1047 \\
27\end{array}$ & $\begin{array}{l}5177 \\
6745\end{array}$ & $\begin{array}{c}23150 \\
0\end{array}$ & $\begin{array}{l}3189 \\
2454\end{array}$ & $\frac{1}{27988}$ & $\begin{array}{l}12613 \\
1634\end{array}$ & $\begin{array}{c}19914 \\
0\end{array}$ & $\begin{array}{l}2861 \\
2027\end{array}$ & 0 \\
\hline$\stackrel{50}{20+30}$ & $\begin{array}{l}\mathrm{MD} \\
\mathrm{FD}\end{array}$ & $\begin{array}{c}0 \\
72\end{array}$ & $\begin{array}{c}27289 \\
0\end{array}$ & $\begin{array}{c}2283 \\
25\end{array}$ & $\begin{array}{l}7930 \\
4981\end{array}$ & $\begin{array}{c}28790 \\
0\end{array}$ & $\begin{array}{l}4632 \\
3165\end{array}$ & $\begin{array}{c}0 \\
34189\end{array}$ & $\begin{array}{c}17740 \\
1016\end{array}$ & $\begin{array}{c}25504 \\
0\end{array}$ & $\begin{array}{l}1831 \\
2309\end{array}$ & 0 \\
\hline $\begin{array}{c}60 \\
25+35 \\
70 \\
40+30\end{array}$ & $\begin{array}{l}\mathrm{MD} \\
\mathrm{FD} \\
\mathrm{MD} \\
\mathrm{FD}\end{array}$ & $\begin{array}{c}0 \\
55 \\
0 \\
41\end{array}$ & $\begin{array}{c}32780 \\
0 \\
38492 \\
0\end{array}$ & $\begin{array}{c}4220 \\
21 \\
7015 \\
49\end{array}$ & $\begin{array}{c}11411 \\
3396 \\
16565 \\
2138\end{array}$ & $\begin{array}{c}34798 \\
0 \\
40632 \\
0\end{array}$ & $\begin{array}{l}6776 \\
3271 \\
9414 \\
2819\end{array}$ & $\begin{array}{c}0 \\
40665 \\
0 \\
46278\end{array}$ & $\begin{array}{c}23200 \\
631 \\
23608 \\
362\end{array}$ & $\begin{array}{c}32504 \\
0 \\
38001 \\
0\end{array}$ & $\begin{array}{l}2468 \\
2622 \\
6932 \\
3450\end{array}$ & 0 \\
\hline $\begin{array}{c}80 \\
35+45\end{array}$ & $\begin{array}{l}\mathrm{MD} \\
\mathrm{FD}\end{array}$ & $\begin{array}{l}0 \\
30\end{array}$ & $\begin{array}{c}44085 \\
0\end{array}$ & $\begin{array}{c}11511 \\
142\end{array}$ & $\begin{array}{c}21090 \\
1212\end{array}$ & $\begin{array}{c}50992 \\
0\end{array}$ & $\begin{array}{c}11594 \\
2001\end{array}$ & $\begin{array}{c}0 \\
46278\end{array}$ & $\begin{array}{c}34704 \\
153\end{array}$ & $\begin{array}{c}44762 \\
0\end{array}$ & $\begin{array}{c}10050 \\
4706\end{array}$ & 0 \\
\hline $\begin{array}{c}90 \\
40+50\end{array}$ & $\begin{array}{l}\mathrm{MD} \\
\mathrm{FD}\end{array}$ & $\begin{array}{c}0 \\
18\end{array}$ & $\begin{array}{c}49588 \\
0\end{array}$ & $\frac{23317}{562}$ & $\underset{501}{27115}$ & $\begin{array}{c}52343 \\
0\end{array}$ & $\begin{array}{c}14309 \\
1305\end{array}$ & $\begin{array}{c}0 \\
52342\end{array}$ & $\begin{array}{c}40795 \\
66\end{array}$ & $\begin{array}{c}50254 \\
0\end{array}$ & $\begin{array}{c}19581 \\
3859\end{array}$ & 0 \\
\hline
\end{tabular}


Nepal Journal of Science and Technology Vol. 15, No.1 (2014) 67-76

Table 9. Comparitive results for PSNR of Lena image according to noise mdoel 4

\begin{tabular}{|c|c|c|c|c|c|c|c|c|c|c|c|}
\hline \multirow{2}{*}{$\begin{array}{l}\text { Noise } \\
\text { Density, } \\
(\%)\end{array}$} & \multicolumn{11}{|c|}{ Method } \\
\hline & $\mathrm{ABDND}$ & ASMF & $\mathrm{BDND}$ & Fuzzy & IASMF & Lapiacian & MASMF & $\begin{array}{l}\text { Mhin- } \\
\text { Max }\end{array}$ & PSMF & PSMF & $\begin{array}{l}\text { Prop } \\
\text { osed }\end{array}$ \\
\hline $\begin{array}{l}10 \\
6+4\end{array}$ & $37-33$ & $17-24$ & $37-39$ & $26-59$ & 16.23 & 31.78 & 1780 & 21.05 & 20.18 & 27.69 & 38.89 \\
\hline $\begin{array}{l}20 \\
8+12\end{array}$ & 3431 & 1268 & 34.30 & 20.32 & 12.56 & 27.78 & 1360 & 15.93 & 14.29 & 27.15 & 35.81 \\
\hline $\begin{array}{l}30 \\
10+20\end{array}$ & 32.17 & 11.70 & 27.38 & 1607 & 11.71 & 2181 & 1155 & 12.92 & 12.02 & 26.17 & 33.67 \\
\hline $\begin{array}{l}40 \\
25+15\end{array}$ & 30.28 & 10.15 & 26.11 & 13.61 & 10.26 & 18.19 & 10.10 & 11.11 & 10.38 & 25.03 & 31.78 \\
\hline $\begin{array}{l}50 \\
20+30\end{array}$ & 28.71 & 8.27 & 23.40 & 11.17 & 8.55 & 15.48 & 8.89 & 9.52 & 9.08 & 23.41 & 30.21 \\
\hline $\begin{array}{l}60 \\
25+35\end{array}$ & 27.15 & 7.48 & 20.11 & 9.48 & 7.72 & 1329 & 8.03 & 8.45 & 8.16 & 20.29 & 28.65 \\
\hline $\begin{array}{l}70 \\
40+30\end{array}$ & 25.86 & 7.33 & 15.48 & 8.48 & 7.54 & 1106 & 7.42 & 7.63 & 7.45 & 15.65 & 27.36 \\
\hline $\begin{array}{l}80 \\
35+45\end{array}$ & 24.23 & 6.21 & 11.40 & 7.21 & 6.39 & 8.94 & 6.71 & 6.80 & 6.77 & 10.42 & 25.73 \\
\hline 90 & 21.81 & 5.74 & 7.42 & 6.39 & 5.85 & 7.41 & 6.18 & 6.21 & 6.18 & 6.98 & 23.31 \\
\hline
\end{tabular}

It can be clearly noticed that the pertormance of ABDND is well for all impulse noise models and all noise densities with almost zero MD and low FD, ASMF gives zero MD and FD for impulse noise models 1 and 2 but MD is very large for impulse noise models 3 and 4. BDND performs well for impulse noise models 1 and 2 with low MD and FD but for impulse noise models 3 and 4 its performance decreases at high noise densities and MD gets very large values. Fuzzy detector comes up with low MD and large FD for impulse noise models 1 and 2 and for impulse noise models 3 and 4 with very large both of MD and FD at all noise densities. IASMF shows results very similar to those of ASMF. Laplacian detector increases the MD and FD for all impulse noise models as the noise density increases. Performance of MASMF is good for impulse noise models 1 and 2 but gives large MD for impulse noise models 3 and 4. For impulse noise models 1 and 2 Min-Max detector gives zero MD and FD but for impulse noise models 3 and 4 shows very large MD. MRD shows zero MD, small FD for impulse noise models 1 and 2 and very large MD, very low FD for impulse noise models 3 and 4 at all noise densities.
PSMF increases the MD and FD with increase in noise densities for all impulse noise models.

Performance of proposed method is very well for all impulse noise models with almost zero MD and FD for all impulse noise models and highest PSNR values. A pixel contaminated by any impulse noise has a relatively high intensity value from its neighborhood. To check whether a pixel is contaminated or not, its distance is measured from median and mean of its neighborhood. After experimenting two threshold values 20 and 30 have been decided for accurate detection because for correct noise cancellation accurate detection is necessary. Proposed filtration or restoration process efficiently reduces the noise by using the median of reference values generated neighborhood. To visualize the qualitative performance of proposed approach restored images have been given in Figure 3, the images of 'Lena', 'Baboon' and 'peppers' have been corrupted by 'salt and pepper' noise according to impulse noise model 1 at $60 \%$ of noise density. Restored images by proposed method have been shown correspondingly. 


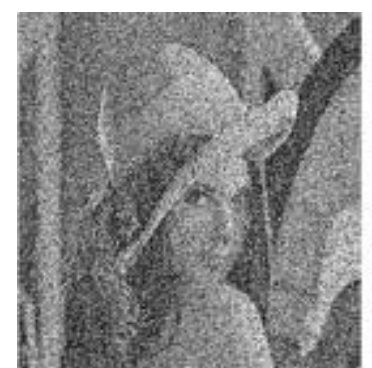

(a)

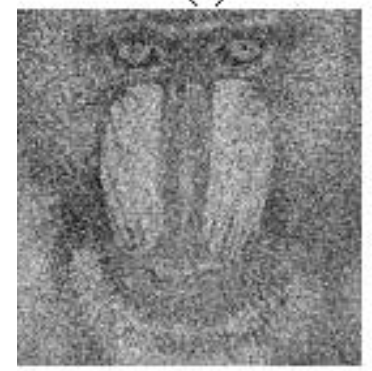

(c)

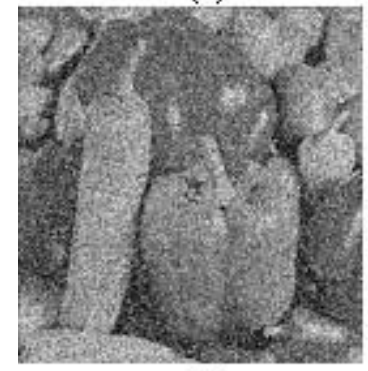

(e)

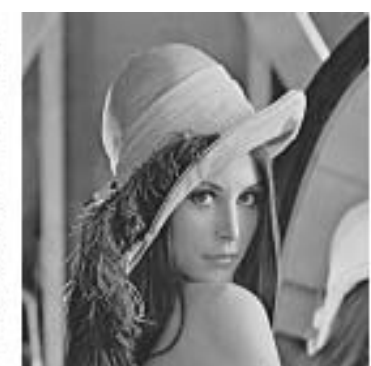

(b)

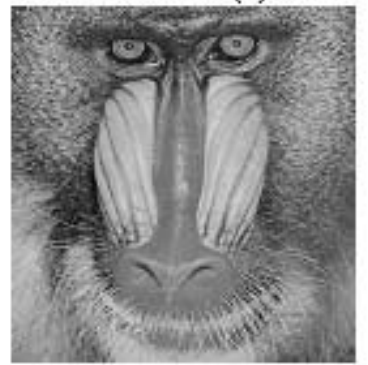

(d)

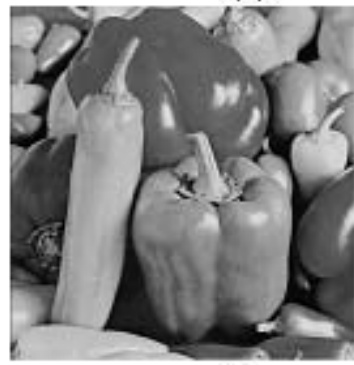

(f)
Fig.3. Images corrupted by $60 \%$ of impulse noise according to noise model 1

(a) 'Lena' image corrupted by $40 \%$ impulse noise'

(b) 'Lena' image restored by proposed method

(c) 'Baboon' image corrupted by $40 \%$ impulse noise'

(d) 'Baboon' image restored by proposed method

(e) 'Peppers' image corrupted by $40 \%$ impulse noise'

(f) 'Peppers' image restored by proposed method

An accurate and efficient impulse noise detection and reduction method has been proposed in this study consisting of two stages; impulse noise detection and filtration. The special property of the method is that it detects a pixel as contaminated and performs noise cancellation process at that moment as a result of which a contaminated pixel is never found in pre neighborhood of a contaminated pixel. Enough uncorrupted pixels are found to estimate the intensity of original image for replacing the contaminated image even in case of very high noise densities (up to 90\%). Comparative experimental results reveal the outperformance of proposed approach over the various existing approaches to noise detection and cancellation. Another fact has also been revealed that a good noise cancellation depends upon the accurate noise detection.

\section{References}

Chan, R. H., C. Hu et al. 2004. An iterative procedure for removing random-valued impulse noise. Signal Processing Letters, IEEE 11: 921-924.

Chen, T. and H. R. Wu. 2001. Adaptive impulse detection using center-weighted median filters. Signal Processing Letters, IEEE 8: 1-3.

Eng, H.L. and K.K. Ma. 2001. Noise adaptive soft-switching median filter. Image Processing, IEEE Transactions on 10: 242-251.

Garnett, R., T. Huegerich et al. 2005. A universal noise removal algorithm with an impulse detector. Image Processing, IEEE Transactions on 14: 1747-1754.

Lee, Y., N. Takahashi et al. 2007. Adaptive partial median filter for early CT signs of acute cerebral infarction. International Journal of Computer Assisted Radiology and Surgery 2: 105-115.

Luo, W. 2007. An efficient algorithm for the removal of impulse noise from corrupted images. $A E U$ International Journal of Electronics and Communications 61: 551-555.

Nallaperumal, K. and J. Varghese et al. 2006. Iterative adaptive switching median filter. Industrial Electronics and Applications 2006 1ST IEEE Conference, pp. 1-6.

Nallaperumal, K., J. Varghese et al. 2007. Salt \& pepper impulse noise removal using adaptive switching median filter. OCEANS 2006-Asia Pacific, pp. 1-8.

$\mathrm{Ng}$, P.E. and K.K. Ma. 2006. A switching median filter with boundary discriminative noise detection for extremely corrupted images. Image Processing, IEEE Transactions 15: 1506-1516.

Toprak, A. and Ý. Güler. 2006. Suppression of impulse noise in medical images with the use of fuzzy adaptive median filter. Journal of Medical Systems 30: $465-471$.

Tripathi, A. and U. Ghanekar et al. 2011. Switching median filter: advanced boundary discriminative noise detection algorithm. Image Processing, IET 5: s598-610.

Vijaykumar, V. and P. Vanathi et al. 2008. High density impulse noise removal using robust estimation based filter. IAENG International Journal of Computer Science 35.

Wang, Z. and D. Zhang. 1999. Progressive switching median filter for the removal of impulse noise from highly corrupted images. Circuits and Systems II: Analog and Digital Signal Processing, IEEE Transactions on 46: $78-80$. 
Ze-Feng, D. and Y. Zhou-Ping et al. 2007. High probability impulse noise-removing algorithm based on mathematical morphology. Signal Processing Letters, IEEE 14(1): 31-34

Zhang, S. and M. A. Karim. 2002. A new impulse detector for switching median filters. Signal Processing Letters, IEEE 9: 360-363.

Zhang, X. and Z. Yin et al. 2008. Adaptive switching mean filter for impulse noise. Congress on image and signal processing, CISP 3: 275-278.
Zvonarev, P. S. and I. V. Apalkov et al. 2005. Neural network adaptive switching median filter for the restoration of impulse noise corrupted images. In: Proceedings of second international conference on image analysis and recognition (September 28-30, 2005) Toronto, Canada (Eds. Mohamed Kamel and Aurelio Campilho) Springer: 223-230. 